\title{
Attitude Control System Design for CubeSats Configured with Exo-Brake Parachute
}

\author{
Sean Shan-Min Swei, ${ }^{*}$ Alexander J. Westfall ${ }^{\dagger}$ \\ NASA Ames Research Center, Moffett Field, CA 94035-1000
}

\begin{abstract}
This paper develops a novel attitude control strategy for an Earth orbiting CubeSat spacecraft by utilizing the exo-brake parachute to modulate the atmospheric drag forces as a source of attitude control authority, enabling orbital exo-sail maneuvers. In particular, the spacecraft attitude controls can be realized through the two dimensional exo-sail maneuvers in pitch and yaw directions. The uncertain atmospheric drag induced disturbance torque is estimated through an adaptive parameter estimation process which makes use of the adaptive least-squares minimization techniques. The covariance updating law with a variable forgetting factor is adopted and it can be shown that the convergent rate for the estimation errors can be chosen at the same level as the forgetting factor, in order to meet the design needs. The proposed approach is best suited for Earth orbiting micro/nano-satellite applications, which are configured with exo-brake parachute. With integration of exo-sail actuation mechanism and disturbance estimation, we demonstrate through simulations that exo-sail induced control torque for CubeSat attitude maneuver is feasible.
\end{abstract}

\section{Introduction}

With its standardized form factor, the CubeSats are ideal platform for performing cost effective science missions in space for limited duration. In recent years, however, researchers are starting to explore potential CubeSat application for longer mission life cycle or beyond Low Earth Orbit (LEO) to, for instance, deep space exploration. To realize this objective, a number of technical challenges must be addressed, especially in the area of attitude controls, for CubeSat's compact dimension makes the design and implementation of an effective control system more challenging. Much of the current research has been focusing on regurgitating the conventional means of controlling large spacecraft and scaling it down for small or CubeSat form factor application. The primary uncertainty of this approach is the availability of the miniaturized attitude actuation or momentum management device that is functionally suited for meeting the mission requirements. Figure 1 shows a sample CubeSat known as the TechEdSat, which is a technical education satellite and part of the International Space Station (ISS) missions aimed for technology demonstrations.

Estimation of environmental disturbance input is a subject of practical significance in space application, especially for micro satellites or CubeSats conducting science missions near Earth or Moon orbits subjected to unknown disturbance torques due to, for example, aerodynamic drag, magnetic field, and gravity gradient. Conventionally, these external disturbance torques are taken into consideration when determining the momentum management operations for space mission planning, as these would impact the sizing of the momentum management actuators, such as reaction wheels and reaction control system (RCS) thrusters. For large spacecraft missions, such as Hubble Telescope ${ }^{1}$ or Solar Dynamic Observatory (SDO), ${ }^{2}$ sufficient margins are often built into the design and into the selection of actuators. However, for a micro satellite or CubeSat application, because of its low cost and high risk nature of missions, the amount of science and the level of precision it can attain are limited by the available actuation control authority and the disturbance environment it is exposed to.

NASA is planning to conduct exo-brake technology demo using a series of TechEdSat platform satellites to demonstrate safe reentry missions after jettisoned from the ISS, see Fig. 2. The goal is to use exo-brake

\footnotetext{
*Research Scientist, Intelligent Systems Division, Associate Member.

${ }^{\dagger}$ Aerospace Engineer, Adecco at GoogleResearch.
} 
parachute as a decelerator in exosphere environment to slow down the reentry speed for potential small payload return from LEO. In this paper, we consider an Earth orbiting CubeSat which is configured with exo-brake mechanism, and propose to develop a novel attitude control concept by incorporating an adaptive disturbance estimator. ${ }^{5}$ The objective is to utilize the disturbance torque through adaptive identification and use it for orbital attitude maneuvers as a means of control source. This approach is especially appealing for micro satellite or CubeSat applications, where the impact of disturbance torque is more pronounced because of its limited control authority relative to its size/mass.

This paper is organized as follows. Section II presents an introduction of spacecraft dynamics including the quaternion dynamics. Section III contains the derivation of exo-sail kinematics and shows the computation of empirical atmospheric drag induced exo-sail torque as functions of pitch and yaw angles. In addition, a look-up table is created to be used as an embedded function for later analysis. An adaptive least-squares approach is introduced in Section IV, where we utilize the notion of quadratic stability to demonstrate that the rate of convergence for disturbance estimation error is guaranteed to be the same as the forgetting factor. A standard quaternion-based controller is proposed with the "PD" control gains as tuning variables. Section V illustrates the process we have taken to derive iteratively the possible exo-sail orientations based on commanded torques. Analyses and simulation results are presented in Section VI, where we demonstrate the efficacy of the proposed exo-sail concept. Finally, the paper summaries in Section VII.

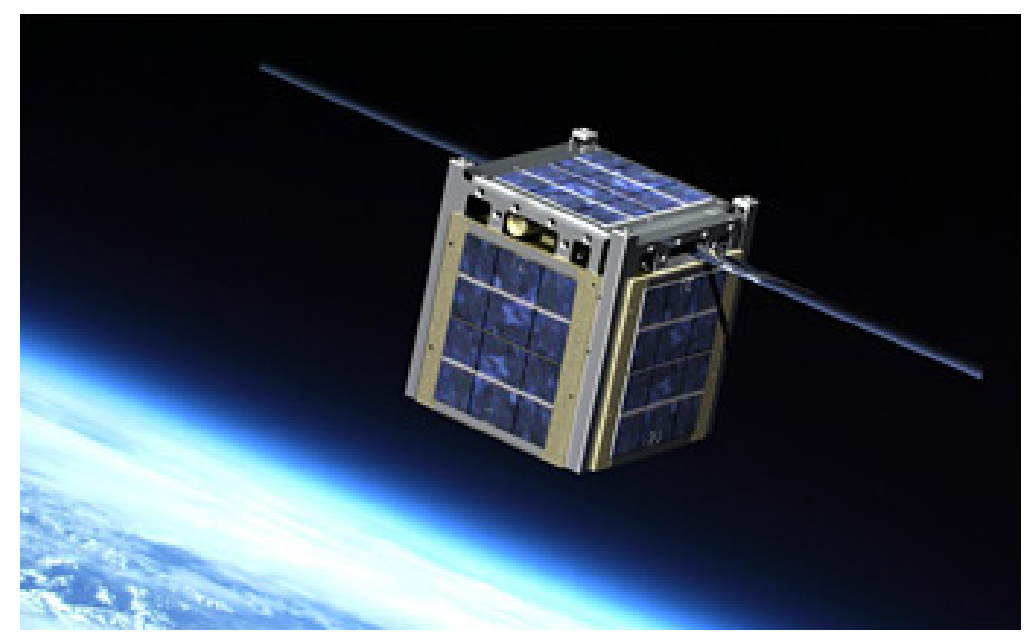

Figure 1. NASA CubeSats mission. ${ }^{3}$

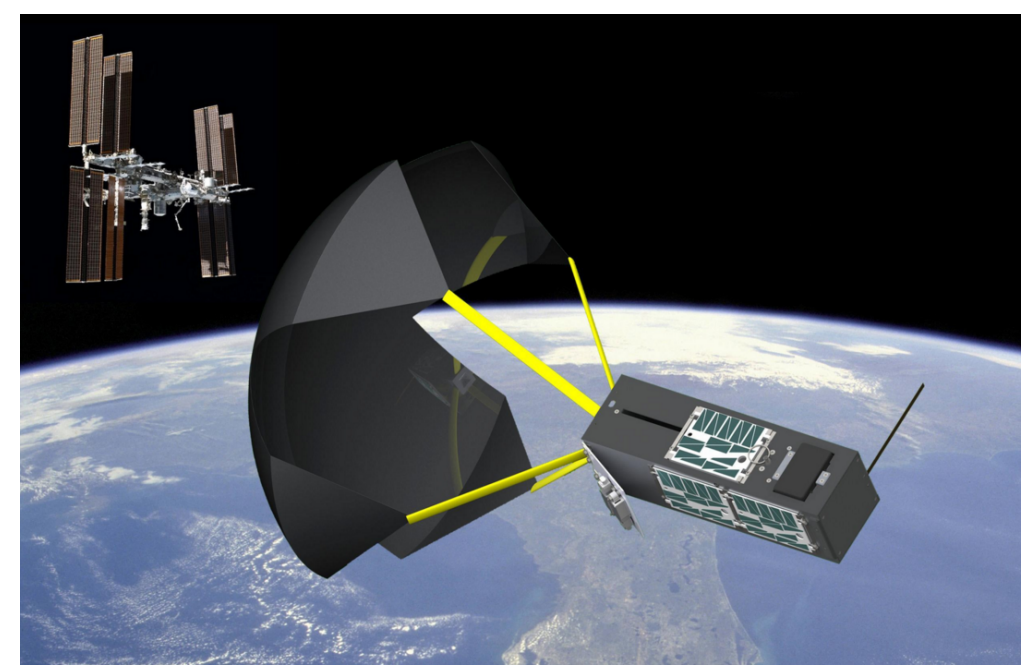

Figure 2. NASA TechEdSat-4 with deployed exo-brake parachute. ${ }^{4}$ 


\section{Spacecraft Dynamics}

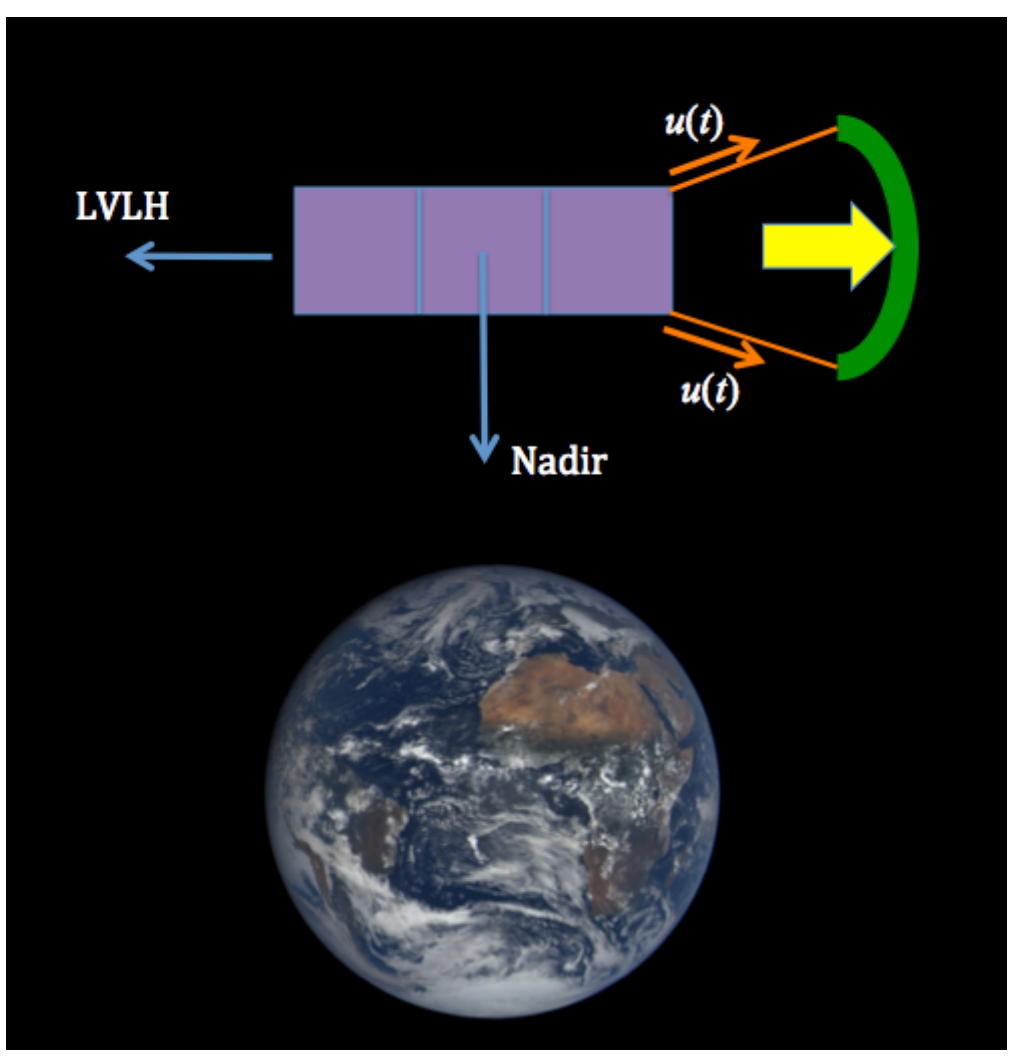

Figure 3. An orbiting 3U CubeSat with exo-brake.

The angular momentum for the orbiting spacecraft in the inertial frame is given by

$$
{ }^{I} H^{s s}=J^{I} \omega^{b},
$$

where $J$ is the mass moment of inertia of the spacecraft, excluding the exo-brake parachute, and ${ }^{I} \omega^{b}=$ $\left[\begin{array}{lll}\omega_{x} & \omega_{y} & \omega_{z}\end{array}\right]^{T}$ is the angular velocity of the spacecraft relative to the inertial frame. Let $\tau$ denote the total external torque, by taking the time derivative of Eq. (1) and setting it equal to the external torque, we obtain

$$
\begin{aligned}
\tau & =\frac{d^{I I} H^{s s}}{d t} \\
& =\frac{d^{b I} H^{s s}}{d t}+{ }^{I} \omega^{b} \times{ }^{I} H^{s s} .
\end{aligned}
$$

Substituting Eq. (1) into above yields,

$$
\tau=J^{I} \dot{\omega}^{b}+{ }^{I} \omega^{b} \times J^{I} \omega^{b} .
$$

Let $u=\left[\begin{array}{lll}u_{x} & u_{y} & u_{z}\end{array}\right]^{T}$ be the external control torque generated by the exo-brake, and $f$ the external environmental disturbance torque, then the total external torque can be expressed as

$$
\tau(t)=u(t)+f(t)
$$

therefore, Eq. (3) can be rewritten as

$$
J \dot{\omega}(t)=J \omega(t) \times \omega(t)+u(t)+f(t),
$$

where, for convenience, we have dropped the superscripts. 
The spacecraft attitude dynamics can be expressed in terms of quaternions as follows, ${ }^{6}$

$$
\begin{aligned}
q & =\left[\begin{array}{c}
q_{1} \\
q_{2} \\
q_{3}
\end{array}\right]=\left[\begin{array}{l}
e_{1} \sin (\phi / 2) \\
e_{2} \sin (\phi / 2) \\
e_{3} \sin (\phi / 2)
\end{array}\right], q_{4}=\cos (\phi / 2) \\
\text { or } \quad & \hat{q}=\left[\begin{array}{c}
q \\
q_{4}
\end{array}\right],
\end{aligned}
$$

where $\left(e_{1}, e_{2}, e_{3}\right)$ are the direction cosines of the Euler axis relative to the body-fixed frame, and $\phi$ is the magnitude of the Euler axis rotation. Clearly, from Eq. (5) we can deduce that

$$
q_{1}^{2}+q_{2}^{2}+q_{3}^{2}+q_{4}^{2}=1 \text { or }\|\hat{q}\|^{2}=1 .
$$

The quaternion kinematic differential equations are then given by

$$
\dot{\hat{q}}=\frac{1}{2} \Omega \hat{q}
$$

where $\Omega$ is defined as

$$
\Omega=\left[\begin{array}{cccc}
0 & \omega_{3} & -\omega_{2} & \omega_{1} \\
-\omega_{3} & 0 & \omega_{1} & \omega_{2} \\
\omega_{2} & -\omega_{1} & 0 & \omega_{3} \\
-\omega_{1} & -\omega_{2} & -\omega_{3} & 0
\end{array}\right]
$$

Equivalently, Eq. (6) can be rewritten as follows,

$$
2 \dot{\hat{q}}=\left[\begin{array}{c}
Q \\
-q^{t}
\end{array}\right] \omega ; Q=\left[\begin{array}{ccc}
q_{4} & -q_{3} & q_{2} \\
q_{3} & q_{4} & -q_{1} \\
-q_{2} & q_{1} & q_{4}
\end{array}\right] .
$$

Recall that $H=J \omega$ is the spacecraft momentum, then we can express (4) in terms of $H$, and along with (6), we now have a complete equation of motion for spacecraft attitude control during orbital maneuvers,

$$
\Sigma:\left\{\begin{array}{l}
\dot{H}(t)=H(t) \times \omega(t)+u(t)+f(t) \\
\dot{\hat{q}}(t)=\frac{1}{2} \Omega(t) \hat{q}(t)
\end{array}\right.
$$

This paper is to design an estimator for disturbance torque $f(t)$, therefore it can be used to assist the control $u(t)$ for spacecraft attitude maneuvers. We first propose to utilize adaptive least-squares minimization approach to approximate the environmental disturbance torque using a set of orthogonal regression functions, and then integrate it with the feedback estimator for momentum estimation. The attitude (quaternions) measurement data will be recorded and used to profile the disturbance torque $f(t)$, and can be used to characterize its relation to the spacecraft attitude. The role of control $u(t)$ is two folds. First, the tendons that connect to the exo-sail parachute is instrumented so that it can measure the induced torque due to aerodynamic drag, and second, the drag-induced torque can then be modulated for spacecraft attitude corrections or maneuvers.

\section{Attitude Control Using Exo-sail}

In this section, we derive the spacecraft attitude control torque generated by modulating the exo-sail orientations with respect to the drag force. Furthermore, it is assumed that the relevant atmospheric parameters be known at a given flight profile, so we can compute the induced aerodynamic forces and moments inserted onto the exo-sail parachute, as a result the reaction torque exerted onto the spacecraft can be used for its on-orbit attitude maneuvers. In Section IV, we will integrate the atmospheric disturbance estimator into the spacecraft attitude control design. 


\section{III.A. Kinematics of exo-sail}

The orientation of exo-sail can be described using two sets of rotational transformation which relate the exo-sail body-fixed frame to exo-sail control frame. Transformations are made utilizing the same technique as the familiar Euler angle rotations. That is to say, the two degrees of freedom that is used by the exo-sail can be described as a pitching and yawing angle of the exo-sail surface with respect to the inertial frame. The exo-sail body-fixed frame is a reference frame that is located at the center of the exo-sail and is assumed to trail directly behind the center of mass of the CubeSat in the anti-RAM direction. Trailing distance, $A$, is another design parameter, since it can be set to define the length of moment arm.

The exo-sail orientation can be defined through a 2-3 Euler rotation, that is, it is first rotated about the $\mathrm{Y}$-axis by a pitch angle $\beta$, followed by a Z-axis rotation of a yaw angle $\gamma$. The direction cosine matrices for the first and second rotation are described by

$$
R_{\beta}=\left[\begin{array}{ccc}
\cos \beta & 0 & -\sin \beta \\
0 & 1 & 0 \\
\sin \beta & 0 & \cos \beta
\end{array}\right] ; R_{\gamma}=\left[\begin{array}{ccc}
\cos \gamma & \sin \gamma & 0 \\
-\sin \gamma & \cos \gamma & 0 \\
0 & 0 & 1
\end{array}\right]
$$

It should be noted that the pitching and yawing torques generated by the exo-sail can be modulated through varying $\beta$ and $\gamma$ angles. Figure 4 illustrates the exo-sail orientations in pitch and yaw directions.

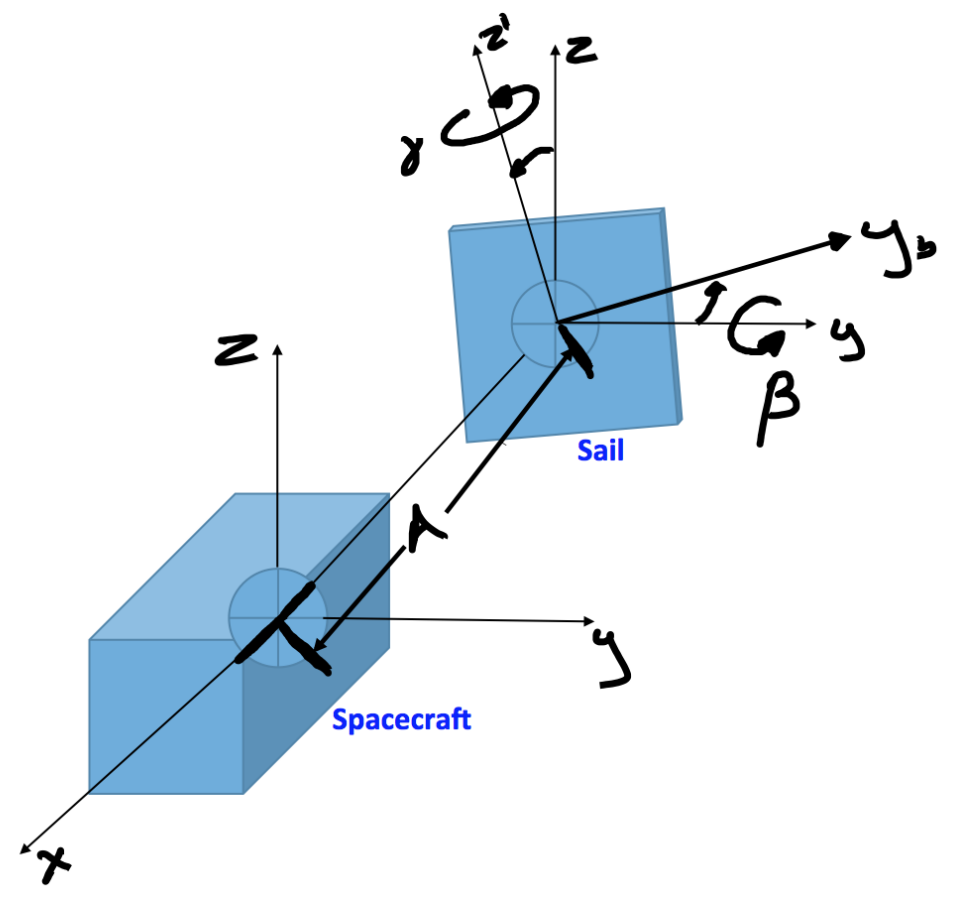

Figure 4. Sail orientations in pitch $(\beta)$ and yaw $(\gamma)$.

In this study, the exo-sail parachute is modeled as a set of co-planar four corner points, and the control transformation can be represented as a set of four vectors in the sail control frame as follows,

$$
r_{1}=\left[\begin{array}{c}
0 \\
s_{y} \\
s_{z}
\end{array}\right], r_{2}=\left[\begin{array}{c}
0 \\
-s_{y} \\
s_{z}
\end{array}\right], r_{3}=\left[\begin{array}{c}
0 \\
s_{y} \\
-s_{z}
\end{array}\right], r_{4}=\left[\begin{array}{c}
0 \\
-s_{y} \\
-s_{z}
\end{array}\right] \text {. }
$$

Furthermore, $r_{i}$ can be transformed from sail control frame to inertial frame through rotations given in (9), and they are described by

$$
\bar{r}_{i}=R_{\gamma} R_{\beta} r_{i}, i=1,2,3,4 .
$$

Since the exo-sail parachute is located some distance from the center of mass of the CubeSat, the vector 
distance between the sail center and the combined center of mass of sail and CubeSat can be defined as

$$
r_{A}=\left[\begin{array}{c}
-A \\
0 \\
0
\end{array}\right]
$$

Now, locating the sail attachment points on the CubeSat, as a set of points some distance from the center of mass of the CubeSat, these attachment points can be described with respect to the CubeSat body-fixed frame as

$$
r_{s 1}=\left[\begin{array}{c}
-x_{1} \\
y_{1} \\
z_{1}
\end{array}\right], r_{s 2}=\left[\begin{array}{c}
-x_{2} \\
-y_{2} \\
z_{1}
\end{array}\right], r_{s 3}=\left[\begin{array}{c}
-x_{3} \\
y_{3} \\
-z_{1}
\end{array}\right], r_{s 4}=\left[\begin{array}{l}
-x_{4} \\
-y_{4} \\
-z_{1}
\end{array}\right] .
$$

Because the sail deflection angles are physically controlled by manipulating tether lengths, it is important to derive each tether as a vector in the CubeSat body-fixed frame, and it is given by

$$
\begin{aligned}
& L_{1}=R_{\gamma} R_{\beta} r_{1}+r_{A}+r_{s 1} \\
& L_{2}=R_{\gamma} R_{\beta} r_{2}+r_{A}+r_{s 2} \\
& L_{3}=R_{\gamma} R_{\beta} r_{3}+r_{A}+r_{s 3} \\
& L_{4}=R_{\gamma} R_{\beta} r_{4}+r_{A}+r_{s 4}
\end{aligned}
$$

and each tether length is the magnitude of its vector, i.e. $\left\|L_{i}\right\|$.

\section{III.B. Exo-sail torque computation}

When the rarified gas interacts with spacecraft, momentum is exchanged. Assuming completely elastic collisions, the particles will reflect off the spacecraft surface in some new direction. The components of this momentum change can be thought of as drag and lift. While the drag component is intuitive to many spacecraft trajectory models, the lifting forces are not. Because they are small and atmospheric interactions are not always well known, most aerodynamic models assume that these forces tend to be considered a source of noise to be compensated for. The aerodynamic forces perpendicular to the body are the essential source of control utilized in this paper. Hastings and Garrett ${ }^{7}$ presented the notion of lifting force, where they express the lifting force, $F_{l}$, for a flat plate at some angle of attack as a function of the atmospheric properties, spacecraft properties, and orientation to the oncoming flow, as follows

$$
F_{l}=v^{2} n m_{p} A_{s} \sin \alpha \sin \theta \cos \theta[(1-\sigma)+(1-\tau)],
$$

where $v$ is the combined speed of spacecraft and oncoming flow, $n$ the number density, $m_{p}$ the average mass of the particles in the flow, $A_{s}$ the surface area of the spacecraft, $\alpha$ the angle of attack of the surface (perpendicular to the drag direction), $\theta$ the angle between the oncoming flow and the vector normal to the spacecraft, $\sigma$ and $\tau$ are accommodation coefficients, which describe how the flow interacts with the spacecraft surface. Continuing the assumption of perfectly elastic collisions, which is to say that the particle momentum reflects perfectly secularly from the surface, and noting that $\sigma=\tau=0$ and $\alpha=\theta-90^{\circ}$, then the lift function (10) simplifies to

$$
F_{l}=-0.25 v^{2} n m_{p} A_{s} \sin \alpha \sin 2 \alpha .
$$

While Hastings and Garrett's model is a 2-dimensional, it can be further expanded into three dimensions by decomposing the lift function into two perpendicular elements that are both perpendicular to the drag. These directions are conveniently chosen to be the pitch and yaw directions, which are perpendicular to the roll (drag) direction. The lift functions can then be written in terms of forces in pitch and yaw directions as follows,

$$
\left\{\begin{array}{l}
F_{\beta}=-0.25 v^{2} n m_{p} A_{s} \sin \beta \cos \gamma \sin 2 \beta \\
F_{\gamma}=-0.25 v^{2} n m_{p} A_{s} \cos \beta \sin \gamma \sin 2 \gamma
\end{array}\right.
$$

where $\beta$ denotes the pitch angle and $\gamma$ the yaw angle. Hastings and Garret also presented statistical measurements for $n$ and $m_{p}$ from the International Space Station (ISS), ${ }^{7}$ and they were given by: $n=10^{12} \mathrm{~m}^{-3}$, $m_{p}=14.3$ (atomic mass unit). These values are also adapted in our simulation study. 
As we indicated earlier, the center of mass of the sail is assumed to stay directly behind the center of mass of the spacecraft, and this allows the moment arm of the sail lifting forces to be at some trailing distance, $A$, of the sail. Therefore, the resultant pitching and yawing torque generated by the lifting forces can simply be described by

$$
\left\{\begin{array}{l}
T_{\beta}=-0.25 v^{2} n m_{p} A_{s} A \sin \beta \cos \gamma \sin 2 \beta \\
T_{\gamma}=-0.25 v^{2} n m_{p} A_{s} A \cos \beta \sin \gamma \sin 2 \gamma
\end{array}\right.
$$

In order to assess and understand the available exo-sail torque control authority, we first need to mesh out the pitch and yaw torques as functions of allowable pitch $(\beta)$ and yaw $(\gamma)$ angles. These meshes will serve as look-up tables so that for any given pair of commanded attitude control torque $\left(T_{\beta}, T_{\gamma}\right)$, a corresponding pair of commanded sail pitch and yaw angles $(\beta, \gamma)$ can be extracted from interpolating the meshes. Figure 5 shows the pitch and yaw control torques generated by the sail parachute as functions of pitch and yaw angles. In theory, however, $\beta$ and $\gamma$ should be directly derived by solving the coupled nonlinear equations (13).

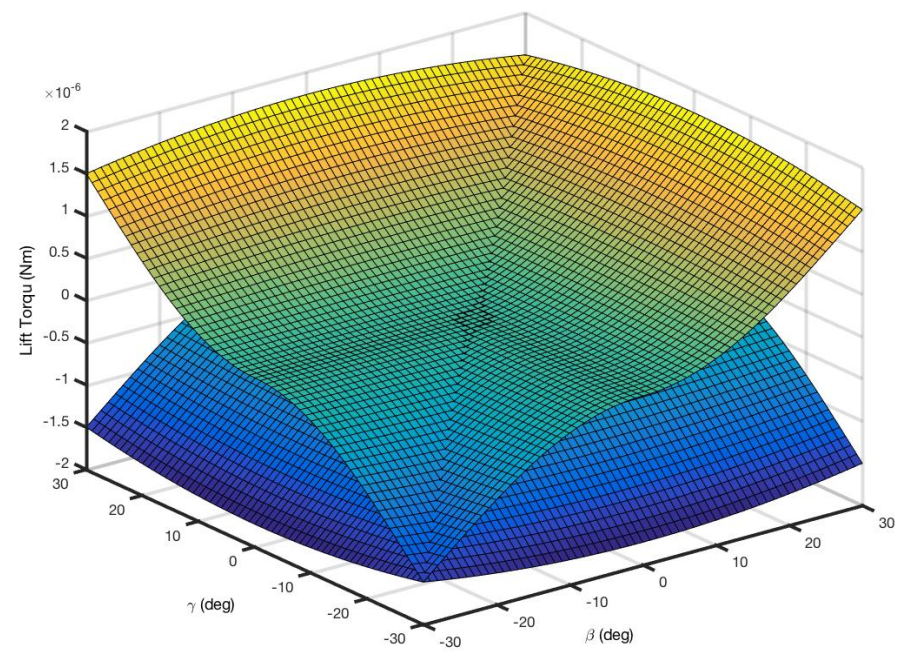

Figure 5. Available control torques as functions of exo-sail orientations.

\section{Adaptive Disturbance Torque Estimation and Controls}

Atmospheric drag-induced disturbance torque is considered dominant source of environmental disturbance for orbiting spacecraft at LEO. In order to characterize this disturbance torque, an adaptive estimator is designed following the process proposed in Swei and Nhan. ${ }^{5}$ The estimator is used to predict aerodynamic force in RAM direction, and once the atmospheric force is determined, it can be applied to the exo-sail, which can then be actively modulated to manipulate the spacecraft attitude. For completeness, we include here the adaptive disturbance torque estimation process.

Recall Eq. (8). Let the disturbance input $f(t)$ be given by

$$
f(t)=\Theta^{T} \Phi(t)
$$

where $\Theta$ is an unknown constant matrix and $\Phi(t)$ a known vector of basis functions, such as Chebyshev polynomials. Let $\Phi(t)=\left[\phi_{1}(t), \phi_{2}(t), \cdots, \phi_{q}(t)\right]^{T}$ where $\left\{\phi_{1}(t), \phi_{2}(t), \cdots, \phi_{q}(t)\right\}$ are linearly independent. Let $\Delta(t)$ be defined as

$$
\Delta(t)=\int_{t_{0}}^{t} \Phi(\tau) \Phi^{T}(\tau) d \tau
$$

and it was proved that the Persistent Excitation $\Delta(t)$ is a positive definite symmetric matrix function. ${ }^{5,8}$ 
Let $\hat{f}(t)$ be an estimate of $f(t)$ at time $t$ and given by

$$
\hat{f}(t)=\hat{\Theta}^{T}(t) \Phi(\tau), t_{0} \leq \tau \leq t,
$$

where $\hat{\Theta}(t)$ is an estimate of $\Theta$ at time $t$. Then, the approximation error $\epsilon(t)$ can be defined by

$$
\epsilon(t)=\hat{\Theta}^{T}(t) \Phi(\tau)-f(\tau), t_{0} \leq \tau \leq t .
$$

Given the approximation error at time $t$, we consider the following exponential cost function,

$$
J_{\epsilon}(\hat{\Theta}(t))=\frac{1}{2} \int_{t_{0}}^{t} e^{-2 \alpha(t-\tau)} \epsilon^{T}(\tau) \epsilon(\tau) d \tau,
$$

where $\alpha \geq 0$ is known as the forgetting factor. ${ }^{9}$ Note that $J_{\epsilon}(\hat{\Theta})$ is a convex function of $\hat{\Theta}$ and that the exponential factor $\alpha$ is imposed to ensure the specified rate of decay for approximation error $\epsilon{ }^{10}$ The minimum for $J_{\epsilon}(\hat{\Theta})$ can be determined from

$$
\frac{\partial J_{\epsilon}^{T}}{\partial \hat{\Theta}^{T}}=0=\int_{t_{0}}^{t} e^{-2 \alpha(t-\tau)} \Phi(\tau) \Phi^{T}(\tau) d \tau \hat{\Theta}(t)-\int_{t_{0}}^{t} e^{-2 \alpha(t-\tau)} \Phi(\tau) f^{T}(\tau) d \tau,
$$

and $\hat{\Theta}(t)$ at time $t$ is obtained as

$$
\hat{\Theta}(t)=R(t) \int_{t_{0}}^{t} e^{-2 \alpha(t-\tau)} \Phi(\tau) f^{T}(\tau) d \tau
$$

where

$$
R(t)=\left[\int_{t_{0}}^{t} e^{-2 \alpha(t-\tau)} \Phi(\tau) \Phi^{T}(\tau) d \tau\right]^{-1} .
$$

It follows readily from (15) that $R(t)>0$. Furthermore, by utilizing the fact that $R(t) R^{-1}(t)=I$ and taking the time derivative on both sides, yields

$$
\frac{d R^{-1}(t)}{d t}=-R^{-1}(t) \dot{R}(t) R^{-1}(t),
$$

where $R^{-1}(t)$ is given by

$$
R^{-1}(t)=\int_{t_{0}}^{t} e^{-2 \alpha(t-\tau)} \Phi(\tau) \Phi^{T}(\tau) d \tau .
$$

To derive the expression for $\frac{d R^{-1}(t)}{d t}$, we make use of the Leibniz's rule ${ }^{11}$ to obtain

$$
\begin{aligned}
\frac{d R^{-1}(t)}{d t} & =\frac{d}{d t} \int_{t_{0}}^{t} e^{-2 \alpha(t-\tau)} \Phi(\tau) \Phi^{T}(\tau) d \tau \\
& =-2 \alpha \int_{t_{0}}^{t} e^{-2 \alpha(t-\tau)} \Phi(\tau) \Phi^{T}(\tau) d \tau+\Phi(t) \Phi^{T}(t) \\
& =-2 \alpha R^{-1}+\Phi(t) \Phi^{T}(t) .
\end{aligned}
$$

Now, we equate (24) to (22), and pre- and post-multiply by the matrix $R(t)$, we obtain

$$
\dot{R}(t)=2 \alpha R(t)-R(t) \Phi(t) \Phi^{T}(t) R(t) .
$$

Similarly, taking the time derivative for (20) renders

$$
\dot{\hat{\Theta}}(t)=\dot{R}(t) \int_{t_{0}}^{t} e^{-2 \alpha(t-\tau)} \Phi(\tau) f^{T}(\tau) d \tau+R(t) \frac{d}{d t} \int_{t_{0}}^{t} e^{-2 \alpha(t-\tau)} \Phi(\tau) f^{T}(\tau) d \tau .
$$

Substituting (25) for $\dot{R}(t)$ and applying the Leibniz's rule to the second term in (26), to obtain

$$
\dot{\hat{\Theta}}(t)=-R(t) \Phi(t)\left\{\Phi^{T}(t) \hat{\Theta}(t)-f^{T}(t)\right\} .
$$


Therefore, (27) and (25) together describe the estimation dynamics. Let the estimation error $\tilde{\Theta}(t)$ be defined by

$$
\tilde{\Theta}(t)=\hat{\Theta}(t)-\Theta, \dot{\tilde{\Theta}}(t)=\dot{\hat{\Theta}}(t),
$$

then substituting (28) into (27), and combining with (25), yields

$$
\left\{\begin{array}{l}
\dot{\tilde{\Theta}}(t)=-R(t) \Phi(t) \Phi^{T}(t) \tilde{\Theta}(t) \\
\dot{R}(t)=2 \alpha R(t)-R(t) \Phi(t) \Phi^{T}(t) R(t)
\end{array}\right.
$$

Note that Eq. (25) is a time-varying Riccati differential equation (TVRDE). In addition, it was shown in Swei and Nhan ${ }^{5}$ that (29) is quadratically stable with rate of convergence $\alpha .{ }^{10}$ That is, $\tilde{\Theta}(t) \rightarrow 0$, and hence $\epsilon(t) \rightarrow 0$, as $t \rightarrow \infty$.

Many known numerical schemes can be used to solve the TVRDE (25) for a given $\alpha$. For instance, one may first convert the TVRDE into coupled $q(q+1) / 2$ differential equations and use numerical integration scheme to solve these equations for $R(t)$ at each $t$, then check if $R(t)>0$. In practice, it is important to note that the upper bound on $\alpha$ can be numerically determined by solving TVRDE for a positive definite solution, hence the maximum achievable rate of convergence is assumed to be known when a set of basis functions $\Phi(t)$ is known.

\section{IV.A. A momentum estimator}

We propose the following momentum estimator for (8),

$$
\dot{\hat{H}}(t)=\hat{H}(t) \times \omega(t)+u(t)+L_{c}(H-\hat{H})+\hat{f}(t),
$$

where $\hat{H}$ denotes the estimate of $H, \hat{f}$ the estimate of $f$ as defined in (16), and $L_{c}>0$ the estimator gain to be chosen. Let the momentum estimation error be defined as

$$
e(t)=\hat{H}(t)-H(t)
$$

then we can simply obtain

$$
\dot{e}(t)=\dot{\hat{H}}(t)-\dot{H}(t) .
$$

Substituting (8) and (30) into (31) yields the estimation error dynamics as

$$
\dot{e}(t)=e(t) \times \omega(t)-L_{c} e(t)+\tilde{\Theta}^{T}(t) \Phi(t),
$$

where we have applied the fact that $\hat{f}(t)-f(t)=\tilde{\Theta}^{T}(t) \Phi(t)$. It was shown in Swei and Nhan ${ }^{5}$ that both $e(t)$ and $\tilde{\Theta}(t)$ converge to 0 at a rate of convergence $\alpha$.

\section{IV.B. Attitude tracking with quaternion feedback}

Let $\hat{q}_{d}$ be the desired orientation of the spacecraft, where

$$
\hat{q}_{d}=\left[\begin{array}{c}
q_{d} \\
q_{4 d}
\end{array}\right] ; q_{d}=\left[\begin{array}{c}
q_{1 d} \\
q_{2 d} \\
q_{3 d}
\end{array}\right] \text {, and }\left\|\hat{q}_{d}\right\|^{2}=1 .
$$

Let the quaternion error $\hat{q}_{e}$ be defined by the quaternion product of the current quaternion $\hat{q}$ and the desired quaternion, which is given by

$$
\hat{q}_{e}=\hat{q} \otimes \hat{q}_{d}=\left[\begin{array}{cc}
Q_{d}^{t} & -q_{d} \\
q_{d}^{t} & q_{4 d}
\end{array}\right]\left[\begin{array}{c}
q \\
q_{4}
\end{array}\right],
$$

where $\otimes$ denotes the quaternion product and

$$
Q_{d}=\left[\begin{array}{ccc}
q_{4 d} & -q_{3 d} & q_{2 d} \\
q_{3 d} & q_{4 d} & -q_{1 d} \\
-q_{2 d} & q_{1 d} & q_{4 d}
\end{array}\right]
$$


Let

$$
\hat{q}_{e}=\left[\begin{array}{c}
\delta q \\
\delta q_{4}
\end{array}\right] ; \delta q=\left[\begin{array}{c}
\delta q_{1} \\
\delta q_{2} \\
\delta q_{3}
\end{array}\right]
$$

and it can be shown that $\hat{q}_{e}$ is a unit quaternion. Furthermore, we can show that quaternion error $\hat{q}_{e}$ satisfies the same error dynamics as described in Eq. (7), that is

$$
\left[\begin{array}{c}
2 \delta \dot{q} \\
2 \delta \dot{q}_{4}
\end{array}\right]=\left[\begin{array}{c}
\delta Q \\
-\delta q^{t}
\end{array}\right] \omega ; \delta Q=\left[\begin{array}{ccc}
\delta q_{4} & -\delta q_{3} & \delta q_{2} \\
\delta q_{3} & \delta q_{4} & -\delta q_{1} \\
-\delta q_{2} & \delta q_{1} & \delta q_{4}
\end{array}\right] .
$$

In the absence of the external disturbance torque $f$, it can be shown that a feedback controller of the form ${ }^{12}$

$$
u=-K_{d} \omega-\operatorname{sign}\left(\delta q_{4}\right) K_{p} \delta q,
$$

globally asymptotically stabilizes the system described in (8), where $K_{d}>0$ is the rate gain and $K_{p}>0$ the attitude gain. The proposed controller (36) is essentially a "PD" controller with an inner rate-loop driven by an outer attitude-loop.

\section{Embedded Torque Function}

Because the simulation will attempt to solve the nonlinear torque equations (13), simultaneously, for both pitch and yaw directions, an embedded function is built to produce the commanded control torques based on a calculated exo-sail orientation. An iterating loop is utilized to search the reachable space of exo-sail orientation to find torques that are close to the desired resultant torques within a specified tolerance. In this study, we neglect exo-sail dynamics and assume that the exo-sail orientation can be manipulated without delay.

To iteratively access the possible exo-sail orientations, a set of for-loops have been used. A mesh grid is created that spans from -30 to +30 degrees of exo-sail orientation to represent the space of allowable orientations in pitch and yaw. The resolution of the grid is set to be at 0.02 degrees, whose choice is only limited by computational power. Because each element of the grid must be checked, increasing the resolution increases each calculation that must be made by the solver at each time step. As each element of the grid is stepped through, the corresponding torques at that element are captured. The candidate exo-sail orientation must have an error less than the allowable tolerance and have less error than the previous candidate elements. If both criteria are met, the torques and angles are recorded and output back into the control system to drive the spacecraft dynamics. If no candidate orientation is found, the tolerance is relaxed by a factor of two and the search continues. This relaxation can only be allowed to a specified limit, beyond that the functional block would output 0 torque and move on to the next time step.

\section{Numerical Simulations}

In this section, we simulate an orbiting CubeSat spacecraft with exo-sail parachute, which is in a deorbiting trajectory at low earth orbit. A mathematical model is developed in Matlab/SIMULINK environment, which includes the spacecraft dynamics, an adaptive disturbance estimator, and the exo-sail control authority look-up tables. To demonstrate spacecraft attitude maneuverability, a 10-degree pitch and 5-degree yaw maneuvers are commanded. The simulations were run for 1600 seconds; long enough to capture the convergence of the maneuvers, due to a small control torque authority generated by the exo-sail.

\section{VI.A. Assumptions and limitations}

In this study, we assume that the spacecraft is axisymmetric and its principal moment of inertia are: $I_{x x}=$ $0.2507 \mathrm{~kg}-\mathrm{m}^{2}, I_{y y}=0.2507 \mathrm{~kg}-\mathrm{m}^{2}$, and $I_{z z}=0.0136 \mathrm{~kg}-\mathrm{m}^{2}$. As indicated earlier, the near earth atmospheric drag is considered the dominant disturbance source exerted to the exo-sail. For simplicity, we assume that the exo-sail has rigid surface, discounting its flexible effects, and treat the tethers connecting the exo-sail and the spacecraft to be rigid, so that they can precisely and kinematically change the exo-sails orientation relative to the spacecraft. 
The quaternion-based feedback controller given in (36) is tuned such that it would not demand an unachievable torque from the exo-sail modulation. To ensure this, the control gains $K_{p}$ and $K_{d}$ are carefully tuned according to the allowable control torque authority. It should be noted that, for given initial and target attitudes, the quaternion solution may create an attitude trajectory that involves performing roll axis maneuver. While development of a reaction wheel control system is outside the scope of this paper, it is assumed that we have one to stabilize the platform in roll axis. In this paper, the simulation assumes an ideal reaction wheel is being implemented that meets the attitude control needs; that is, any desired rotation in spacecraft's roll axis is effectively controlled. Hence, its dynamics and controls are absent from current study. Interested readers may want to consult $\mathrm{Wie}^{12}$ and $\mathrm{Sidi}^{6}$ for treatment of attitude controls using reaction wheels.

In this study, the second order Chebyshev polynomials of the first kind are used as basis functions, that is $\Phi(t)=\left\{1, t, 2 t^{2}-1\right\}$, for adaptive estimation. We also assume the exo-sail parachute cross section area is $1 \mathrm{~m}^{2}$, the assigned rate of convergence or forgetting factor $\alpha$ is set at 0.1 , and the estimator gain $L_{c}$ is chosen to be 0.25 . Furthermore, the pair of control gains is chosen to be: $K_{p}=2.0 e^{-5}$ and $K_{d}=1.74 e^{-6}$.

\section{VI.B. Simulation results}

Initially, the disturbance estimator has no knowledge of actual atmospheric drag forces. As time elapses, the proposed drag force estimation begins to converge to the actual. Figure 6 presents the first few seconds of the estimation error, and as can be seen the convergence of estimation is quite fast, which demonstrates the effectiveness of the proposed adaptive disturbance estimator.

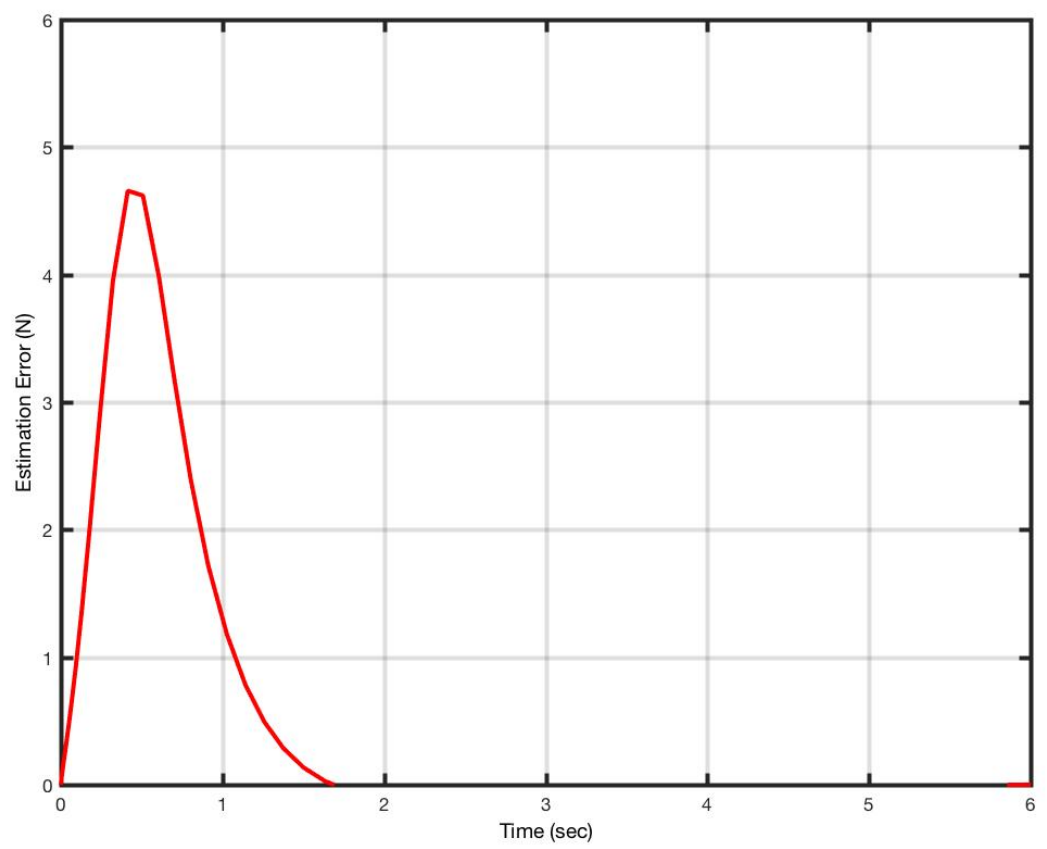

Figure 6. Disturbance drag force estimation error; $\alpha=0.1$.

Figure 7 shows the spacecraft attitude tracking performance; starting from the initial Euler angles $(0$, $0,0)$-deg to the desired attitude $(0,10,5)$-deg, by manipulating the small disturbance torque induced by exo-sail. In addition, Fig. 8 shows the spacecraft's rotational rates.

As noted that the control authority in pitch and yaw axes were generated by the relative orientation of exo-sail's cross section area. Figure 9 shows the control torque created by the exo-sail comparing with the commanded torque from the quaternion error compensator. While an optimal solution for atmospheric drag forces would smoothly transition from one value to the next, instances of discontinuity can be observed. The sudden jumps are locations where the desired torque is not reachable at the given tolerance and mesh resolution in the look-up tables; because if the tolerance is relaxed, a value either above or below the previ- 


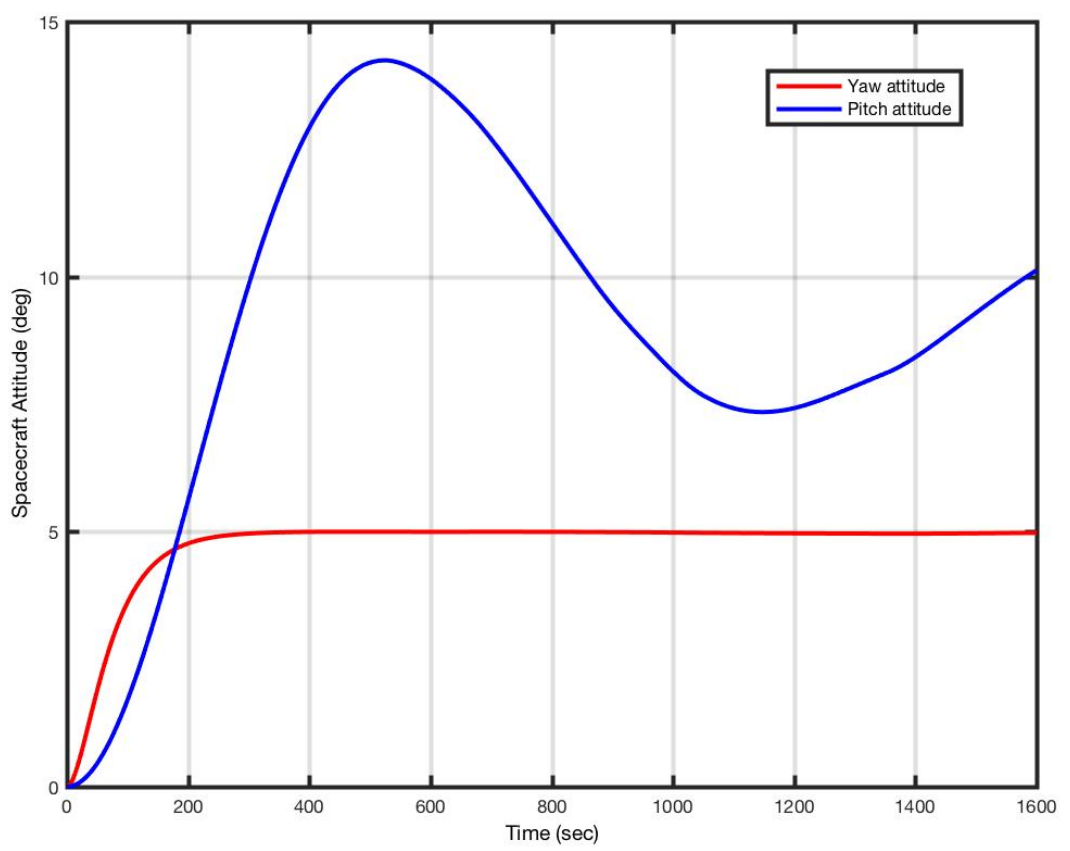

Figure 7. CubeSat attitude tracking performance; from $(0,0,0)$-deg to $(0,10,5)-d e g$.

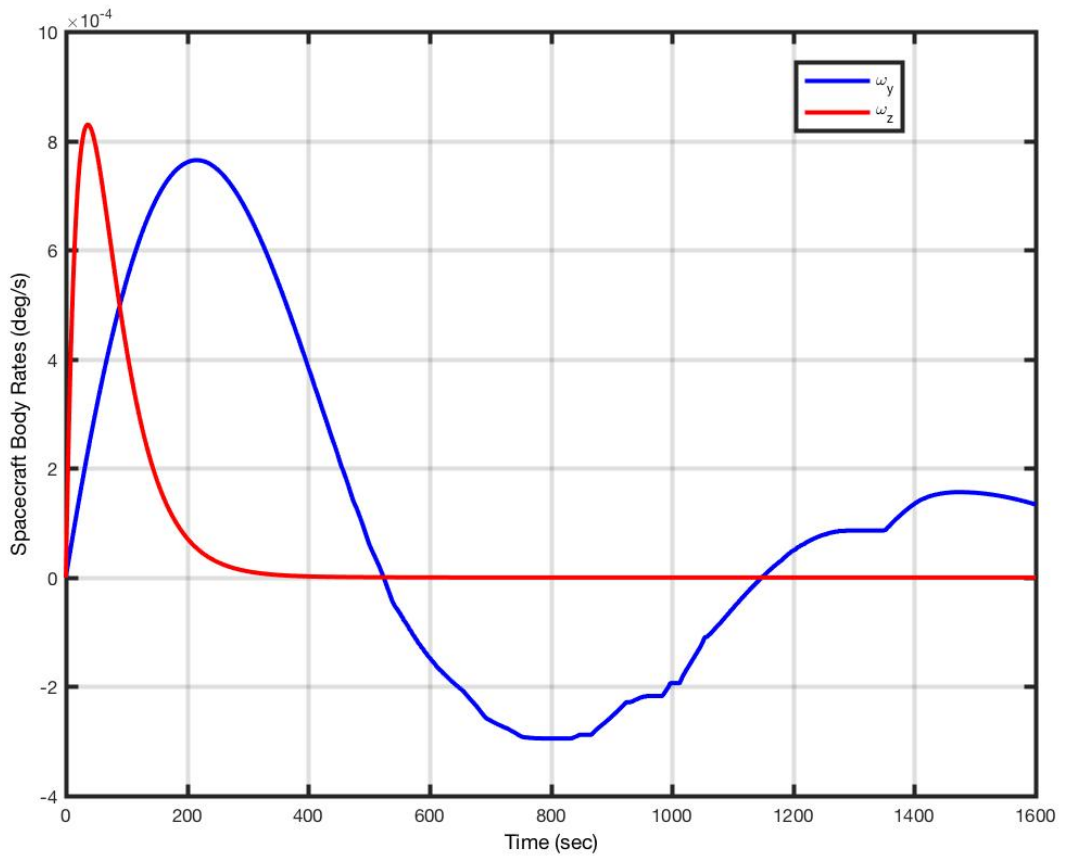

Figure 8. CubeSat rotational rate. 
ously desired torque is selected. The proposed model assumes that the exo-sail orientation is instantaneously reachable, however if the exo-sail dynamics were involved, then the execution to a new orientation would take time to reach, which would smooth out the transition.

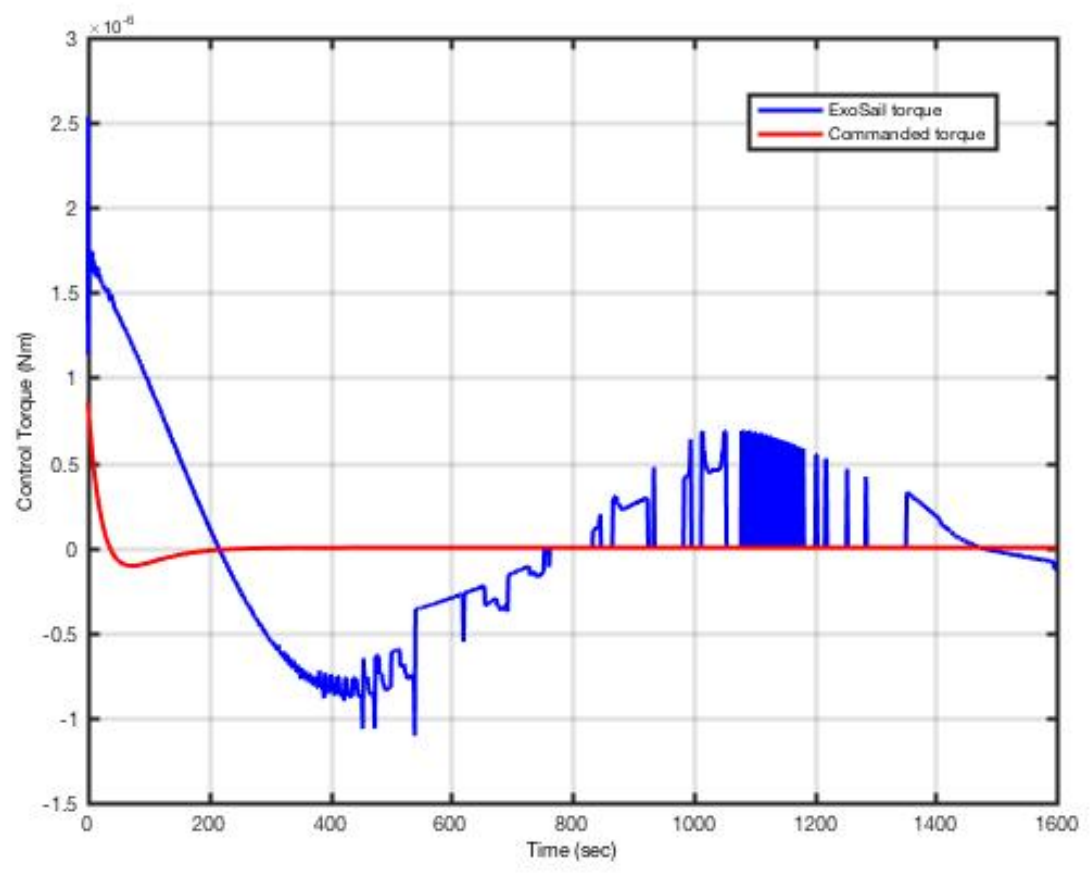

Figure 9. Control torque authority comparison.

\section{Conclusions}

In this paper, we proposed the concept of exo-sail for an Earth orbiting CubeSat spacecraft by utilizing the exo-brake parachute for spacecraft attitude maneuver. The sail kinematics and induced control torque due to atmospheric drag force were studied, and an adaptive disturbance estimator with designated rate of convergence was designed, by integrating the adaptive least-squares method with a momentum estimator. The estimated disturbance aerodynamic forces were then used for spacecraft attitude maneuver. A functional relationship between the exo-sail orientations and the exo-sail induced control torques was established and, for implementation purpose, it was converted into an embedded look-up table. A standard quaternion-based feedback controller was proposed, which was essentially a PD controller with its control gains carefully tuned, so as not to exceed the allowable exo-sail induced control torque. The proposed concept was successfully demonstrated through simulations and the results showed that the proposed spacecraft attitude control strategy using exo-sail was feasible.

\section{References}

\footnotetext{
${ }^{1}$ Markley, F. Landis, and John D. Nelson, "Zero-Gyro Safemode Controller for the Hubble Space Telescope." Journal of Guidance, Control, and Dynamics, Vol. 17, No. 4, 1994, pp. 815-822.

${ }^{2}$ Bourkland, Kristin L., Scott R. Starin, and David J. Mangus, "The Use of a Gyroless Wheel-Tach Controller in SDO Safehold Mode" Flight Mechanics Symposium, 2005.

${ }^{3} \mathrm{http}: / /$ www.nasa.gov/directorates/heo/home/CubeSats_initiative

${ }^{4}$ https://www.nasa.gov/sites/default/files/atoms/files/techedsat4-508-16april2015.pdf

${ }^{5}$ Swei, S.M., Nguyen, N.T., "Adaptive Estimation of Disturbance Torque for Orbiting Spacecraft Using Recursive LeastSquares Methods," AIAA SciTech 2016, San Diego, CA.

${ }^{6}$ Sidi, M.J., Spacecraft Dynamics and Control: An Practical Engineering Approach, Cambridge University Press, 1997.

${ }^{7}$ Hastings, D., Garrett, H., Spacecraft-Environment Interactions, Cambridge University Press, 1996.
} 
${ }^{8}$ Corless, M.J., Frazho, A.E., Linear Systems and Control: An Operator Perspective, Pure and Applied Mathematics, Marcel Dekker, Inc., 2003.

${ }^{9}$ Ioannou, P., Fidan, B., Adaptive Control Tutorial, SIAM, 2006.

${ }^{10}$ Corless, M.J., "Guaranteed Rate of Exponential Convergence for Uncertain Dynamical Systems," Journal of Optimization Theory and Applications, vol. 64, 1990, pp. 481-494.

${ }^{11}$ Anton, H., Calculus with Analytic Geometry, 2nd edition, Wiley, 1984.

${ }^{12}$ Wie, B., Space Vehicle Dynamics and Control, AIAA Education Series, 1998. 